\title{
Disability and Pain are the Best Predictors of Sick Leave After a Distal Radius Fracture in Men
}

\author{
Lisa Egund ${ }^{1,2} \cdot$ Karin Önnby $^{1} \cdot$ Fiona Mcguigan ${ }^{1} \cdot$ Kristina Åkesson $^{1,2}$ (i)
}

Published online: 12 February 2020

(c) The Author(s) 2020

\begin{abstract}
Purpose Distal radius fracture often compromises working ability, but clinical implications are less studied in men due to its lower incidence. This study therefore describes sick leave in men with distal radius fracture, specifically exploring the impact of patient- and fracture-related factors. Methods Professionally active men aged 20-65 with distal radius fracture were followed prospectively for 1-year $(\mathrm{n}=88)$. Data included treatment method, radiographic parameters pre/post treatment, complications, health, lifestyle and occupational demand. Patient outcomes were self-reported sick leave; Disability of the Arm, Shoulder and Hand (DASH) score; pain (5 likert scale); SF-36: Physical Component Scale (PCS) and Mental Component Scale (MCS). Results Median sick leave was 4 weeks (IQR 0; 8); almost a third reported taking no sick leave. Categorizing sick leave into 3 groups (0-6, 7-12 and $>12$ weeks), men with the longest sick leave had 22 points higher DASH score $(\mathrm{p}=0.001)$ and 5 points lower PCS $(\mathrm{p}=0.02)$ at 1 week and the difference remained over time; they were also older and more often treated surgically. The strongest predictors of length of sick leave were one-week post-fracture DASH score $(\mathrm{rs}=0.4, \mathrm{p}<0.001)$, pain intensity $(\mathrm{rs}=0.4, \mathrm{p}<0.001)$ and PCS $(\mathrm{rs}=-0.4, \mathrm{p}=0.002)$. The correlation between sick leave and pain was even stronger analyzing treatment groups separately (closed reduction and cast $r_{s}=0.56, p=0.007$, surgery $\left.\mathrm{r}_{\mathrm{s}}=0.42, \mathrm{p}=0.04\right)$. Conclusions Self-reported disability, pain and global health measurements as early as 1 week post-fracture are the strongest predictors of length of sick leave regardless of treatment; an important finding easily transferrable to clinical management of distal radius fractures.
\end{abstract}

Keywords Distal radius fracture $\cdot$ Men $\cdot$ Sick leave $\cdot$ Outcome $\cdot$ Disability $\cdot$ Pain

\section{Introduction}

The most frequent fracture in adults is the distal radius fracture. The reported incidence rate in Southern Sweden is as high as 278 per 100,000 person years and increasing, especially among those of working age $[1,2]$. Although a distal radius fracture is often regarded as simple with good prognosis, a considerable number of individuals experience prolonged pain and disability [3].

Time lost from work is a common negative consequence after a fracture. Trauma related incapacity is an issue

Kristina Åkesson

kristina.akesson@med.lu.se

1 Department of Clinical Sciences Malmö, Clinical and Molecular Osteoporosis Research Unit, Lund University, Lund, Sweden

2 Department of Orthopedics, Skåne University Hospital, 20502 Malmö, Sweden clinicians deal with every day, although not much studied. Specific factors predictive of length of post-fracture sick leave are still poorly understood; for distal radius fracture only one study has specifically addressed this question [4]. Since men comprise a minority of this fracture population, determinants of sick leave are even less evaluated. From a socio-economic view point, and in the light of an increasing incidence, it is essential to recognize the impact and cost for employers and individuals, as well as where to intervene to reduce this burden.

A commonly held belief is that high work demand and more severe fracture lead to prolonged sick leave $[5,6]$, whereas we hypothesize that patient perception also plays a key role. The aim of the present study is to describe the variation in sick leave among men with distal radius fracture and most importantly to explore the impact of patient-related factors such as self-reported disability and pain, in addition to fracture related variables. 


\section{Methods}

\section{Participants and Design}

We conducted a cohort study of men with distal radius fracture at the Department of Orthopedics, Skåne University Hospital, Malmoe. The main focus was bone mass and risk factors of osteoporosis as earlier described [7]. As part of this larger study, a prospective study was designed to follow patients during 12 months post-fracture and in addition to bone parameters also evaluating functional outcome and sick leave. Consecutive patients with an acute distal radius fracture were invited during 2003-2007. General inclusion criteria: male, age 18-65 years, in active employment or education, acute distal radius fracture and resident in the catchment area. Exclusion criteria were multiple fractures (including bilateral radius fracture), cognitive impairment and insufficiently understanding Swedish to complete the questionnaires.

All parts of the study were approved in advance by the Lund University ethical review board (November 11th 2002, LU 788 02) and performed in compliance with the Helsinki Declaration. All participants gave written informed consent.

We initially identified 333 men aged $18-65$ years with distal radius fracture, of whom 89 did not meet the inclusion criteria (36 with non-acute fracture; 20 with multiple fractures; 10 non-residents, 13 non-Swedish speaking; 10 retired or not in active employment/education). Of the 244 eligible, 88 agreed to participate. Reasons for nonparticipation were general unwillingness (117), illness (18), known substance abuse (14) non-contactable (7). Age distribution of participants and non-participants did not differ.

\section{Treatment Protocol}

Participants were all treated according to established standard treatment protocol for distal radius fracture [8]. Undisplaced or minimally displaced fractures were treated in a short arm cast for 4-5 weeks, unstable fractures were treated with closed reduction and cast and highly unstable fractures were surgically treated (mainly external fixation at the time of investigation). The patients were seen by a physiotherapist within one week after the cast or external fixator removal and again at a final routine check 3-4 weeks later.

\section{Data Collection}

At enrollment, the participants completed a comprehensive questionnaire on health status, medication and life-style factors. Trauma level was recorded-low trauma defined as a fall from standing height or less; high trauma as all other types. Fracture complications within the first year post-fracture were identified retrospectively by reviewing patient records. As a measure of pre-existing comorbidity we calculated Charlson Comorbidity Index (CCI) from the information available in the initial questionnaire.

Socio-economic status (educational level, type of work, workload) was recorded and work demand classified as sedentary, light, medium, heavy, very heavy [9]. Sick leave was self-reported and recorded by questionnaire at 6-8 weeks, 3, 6 and 12 months. In Sweden, the national health insurance allows compensation up to one year when unable to work due to sickness or injury.

\section{Assessment of Disability and Global Health}

The Disability of the Arm, Shoulder and Hand questionnaire (DASH, Swedish version) was recorded at inclusion approximately one week after fracture, and again at 6-8 weeks and 12 months. DASH is a 30 item instrument evaluating disability of the upper limb, which provides an overall score of $0-100$; higher scores indicating higher disability [10]. In addition, in order to further understand the importance of patient perceived pain on sick leave we have specifically analyzed the pain question within the instrument.

As measurements of global health, participants completed the SF-36 questionnaire (1 week; $6-8$ weeks; 12 months) and the EQ-5D-3L (6-8 weeks; 12 months) after fracture.

The SF-36 health status questionnaire has 36 items, producing eight scales in various aspects of physical and mental health, compressible into two overall scores: the physical component score (PCS) and the mental component score (MCS). Each is normalized to a mean of 50 and standard deviation of 10 compared to the general US population, with higher score indicating better quality of life [11].

The EQ-5D-3L consists of the EQ-5D descriptive system and the EQ visual analogue scale, EQ VAS. The latter records the respondent's self-rated health on a vertical, visual analogue scale where the endpoints are labelled 'Best imaginable Health state' and 'Worst imaginable Health state' [12].

\section{Radiographic Evaluation}

Standard postero-anterior and lateral radiographs were obtained at admission to the emergency department and at the routine follow-up 7-10 days later. We evaluated the initial and the latest available radiographic examination measuring dorsal tilt (degrees) and ulnar variance $(\mathrm{mm})$. Dorsal tilt was measured on the lateral view as the angle 
between a line connecting the dorsal and volar lips of the distal radius and a line perpendicular to the central axis of the radius [13]. Ulnar variance was measured on the posteroanterior view by the method of perpendiculars and central reference point $[14,15]$. Radiographs were assessed using Sectra IDS7 version 18.2.18.4066 (Sectra AB, Linkoping Sweden).

\section{Statistics}

Categorical variables are expressed as numbers and continuous variables as mean with standard deviation (SD) and/or range. We used independent unpaired t-test for continuous variables and chi-square for comparisons between categorical variables. DASH data are ordinal with a skewed distribution, therefore non-parametric analyses were employed, and data presented as median (interquartile range, IQR).

Sick leave was analyzed in three clinically relevant groups: 'short' 0-6 weeks (immobilization and two weeks rehabilitation), 'intermediate' 7-12 weeks (allowing for longer rehabilitation) and 'prolonged sick leave' $\geq 13$ weeks. Work demand was compressed into two categories due to the relatively small cohort size: sedentary-medium and heavyvery heavy [16].

To explore associations between specific variables and sick leave category, ANOVA was used for normally distributed, Kruskal-Wallis for non-normally distributed continuous variables and Chi-square for categorical variables. Spearman's rho $\left(r_{s}\right)$ was used for bivariate correlation analysis (which allows inclusion of outliers, nonnormally distributed or non-linearly related variables) and is presented with $95 \%$ confidence interval.

Prior to regression analysis, diagnostics identified three outliers (each with 42 weeks or more of sick leave) which were excluded; residuals from each analysis were thereafter normally distributed. The multiple regression analysis, included age, CCI and work demand (Model 1) i.e. nonmodifiable, non-fracture related factors. Model 2 included age, CCI, work demand and fracture treatment (cast, closed reduction and cast or surgery). To each of these models, disability, pain, or global health were added as explanatory factors. Data is presented as $\mathrm{R}^{2}$ and unstandardized regression coefficient (B), with $95 \%$ confidence interval.

Analyses were performed using SPSS version 25 (IBM Corp., NY, USA). We considered a two-tailed p-value $<0.05$ statistically significant.

\section{Results}

Mean age at fracture was 45 (21-64), (median 48, IQR 33; 57). Twenty-two patients sustained their facture while at work, 25 at sport activities and the remaining at other leisure activities. Almost half of the reported fractures resulted from high energy trauma (Table 1).

Figure 1 shows the distribution of sick leave among the participants which ranged from 0 to 52 weeks (median 4; (IQR 0; 8); mean 6 SD 9). Almost one third (24/88) did not require any sick leave; these men were younger $(p=0.02)$ and had a higher education level $(p=0.002)$. Length of sick leave also differed between non-surgical or surgical treatment (median 4 vs $8, p=0.008$ ). Two had major complications and had not returned to work by the conclusion of the study (one tendon rupture, one displacement requiring surgery within the first year); no cases of Complex Regional Pain Syndrome (CRPS) were reported.

\section{Sick Leave Groups}

To evaluate patient and fracture related factors which might be associated with sick leave, we used three sick leave groups: short, intermediate and prolonged. Men in the prolonged sick-leave group were more likely to have had surgery compared to men with shorter sick leave (Table 1), although timing of surgery had no impact; there was no difference between those who had primary surgery compared to those having secondary surgery due to displacement at the weekly follow-up. There was no difference in length of sick leave in those having AO type A (7 weeks), B (6 weeks) or $\mathrm{C}(6$ weeks) fracture $(\mathrm{p}=0.959)$. Men with the longest sick leave tended to be older with low trauma fractures being more frequent.

Overall, men with the longest sick leave had higher DASH scores and lower PCS at all time-points, although all three groups improved over time. The DASH score at 1 week was 22 points higher in those with prolonged sick leave compared to those with short $(\mathrm{p}=0.001)$ (Table 1 , Fig. 2) and PCS was significantly lower (Table 1, Fig. 3). The difference in DASH score and PCS remained at 12 months post-fracture, most pronounced for PCS. Men with intermediate sick leave and even more pronounced in the prolonged group showed significantly lower EQ5D VAS scores compared to those in the short $(\mathrm{p}=0.008)$ (Table 1$)$.

\section{Predictors of Length of Sick Leave}

Correlations with length of sick leave were then explored; consistent associations were observed with self-reported disability and general health (Table 2). The correlation 
Table 1 Patient- and fracture related factors and disability/global health of all men of working age with distal radius fracture and also by duration of sick leave

\begin{tabular}{|c|c|c|c|c|c|}
\hline & All DRF Patients & Short $0-6$ weeks & $\begin{array}{l}\text { Intermediate } \\
7-12 \text { weeks }\end{array}$ & Prolonged $>12$ weeks & $p$-value ${ }^{\S}$ \\
\hline & $\mathrm{N}=88$ & $\mathrm{~N}=56$ & $\mathrm{~N}=25$ & $\mathrm{~N}=7$ & \\
\hline \multicolumn{6}{|l|}{ A) Patient related factors } \\
\hline Age, years $(\mathrm{SD})$ & $45(14)$ & $43(14)$ & $49(13)$ & $53(10)$ & $0.038^{\text {II }}$ \\
\hline BMI, $\mathrm{kg} / \mathrm{cm}^{2}(\mathrm{SD})$ & $26.1(3.8)$ & $25.3(4.0)$ & $27.5(3.4)$ & $27.1(2.3)$ & $0.065^{\#}$ \\
\hline Charlson Comorbidity Index (SD) & $0.3(0.6)$ & $0.3(0.6)$ & $0.3(0.6)$ & $0.9(1.1)$ & $0.006^{\dagger \dagger}$ \\
\hline Alkohol, units (10 g)/week (SD) & $9(8)$ & $10(8)$ & $8(9)$ & $6(3)$ & $0.245^{\Uparrow \pi}$ \\
\hline \multicolumn{6}{|l|}{ Work status } \\
\hline Professionally active & 82 & 50 & 25 & 7 & \\
\hline Student & 6 & 6 & 0 & 0 & \\
\hline \multicolumn{6}{|l|}{ Educational level } \\
\hline Primary & 17 & 9 & 5 & 3 & $0.089^{\dagger \dagger}$ \\
\hline Secondary & 38 & 21 & 13 & 4 & \\
\hline University & 28 & 23 & 5 & 0 & \\
\hline \multicolumn{6}{|l|}{ Work demand } \\
\hline Sedentary-medium & 72 & 50 & 17 & 5 & $0.066^{\dagger \dagger}$ \\
\hline Heavy-very heavy & 16 & 6 & 8 & 2 & \\
\hline \multicolumn{6}{|l|}{ B) Fracture related factors } \\
\hline Trauma level high & 38 & 24 & 12 & 2 & $0.016^{\dagger \dagger}$ \\
\hline Dominant hand fracture ${ }^{\mathrm{a}}$ & 33 & 23 & 7 & 3 & $0.487^{\dagger \dagger}$ \\
\hline \multicolumn{6}{|l|}{ AO classification } \\
\hline A & 19 & 12 & 4 & 3 & 0.105 \\
\hline B & 11 & 10 & 0 & 1 & \\
\hline $\mathrm{C}$ & 50 & 29 & 18 & 3 & \\
\hline Displacement ${ }^{\beta}$ —initial & $29 / 75$ & $14 / 47$ & $12 / 22$ & $3 / 6$ & $0.121^{\dagger \dagger}$ \\
\hline Displacement-follow up & $4 / 77$ & $2 / 50$ & $2 / 21$ & $0 / 6$ & $0.529^{\dagger \dagger}$ \\
\hline \multicolumn{6}{|l|}{ Treatment } \\
\hline Cast & 42 & 33 & 7 & 2 & $0.024^{\dagger \dagger}$ \\
\hline Closed reduction + cast & 22 & 14 & 6 & 2 & \\
\hline Surgery & 24 & 9 & 12 & 3 & \\
\hline Complication-major & 4 & 1 & 1 & 2 & $0.023^{\dagger \dagger}$ \\
\hline \multicolumn{6}{|l|}{ C) Disability/global health } \\
\hline \multicolumn{6}{|l|}{ DASH (median (IQR)) } \\
\hline 1 week & $41(29 ; 57)$ & $34(23 ; 47)$ & $50(38 ; 59)$ & $56(48 ; 60)$ & $0001^{\mathrm{g}}$ \\
\hline $6-8$ weeks & $14(6 ; 27)$ & $10(4 ; 18)$ & $23(7 ; 31)$ & $27(13 ; 31)$ & $0011^{\text {II }}$ \\
\hline 12 months & $2(0 ; 7)$ & $1(0 ; 3)$ & $6(1 ; 12)$ & $9(8 ; 28)$ & $<0.001^{\mathrm{II}}$ \\
\hline \multicolumn{6}{|l|}{ SF-36 [mean (SD)] } \\
\hline \multicolumn{6}{|l|}{1 week } \\
\hline PCS & $40(6)$ & $41(6)$ & $37(6)$ & $36(3)$ & $0.016^{\#}$ \\
\hline MCS & $48(11)$ & $47(11)$ & $49(12)$ & $46(8)$ & $0.811^{\#}$ \\
\hline \multicolumn{6}{|l|}{$6-8$ weeks } \\
\hline PCS & $43(8)$ & $45(7)$ & $40(8)$ & $38(5)$ & $0.004^{\#}$ \\
\hline MCS & $52(10)$ & $52(9)$ & $52(13)$ & $48(9)$ & $0.582^{\#}$ \\
\hline \multicolumn{6}{|l|}{12 months } \\
\hline PCS & $52(7)$ & $54(6)$ & $51(8)$ & $43(6)$ & $0.001^{\#}$ \\
\hline MCS & $53(6)$ & $53(5)$ & $51(9)$ & $54(8)$ & $0.418^{\#}$ \\
\hline EQ5D VAS 6-8 weeks (mean \pm SD) & $80(13)$ & $83(10)$ & $77(14)$ & $68(23)$ & $0.008^{\#}$ \\
\hline
\end{tabular}

Data is presented as numbers unless otherwise stated and vary slightly due to missing data

${ }^{\mathrm{a}} \mathrm{n}=17$ missing information. ${ }^{\beta}$ Dorsal tilt $>10^{\circ}$ and/or ulnar variance $>2 \mathrm{~mm}$

${ }^{\S} p$-value for observed differences between the 3 sick leave groups. Statistical methods: ${ }^{\mathbb{I}}$ Kruskal-Wallis

"One-way ANOVA

${ }^{\dagger}$ Chi-Square 
Fig. 1 Distribution of weeks of sick leave after distal radius fracture in men

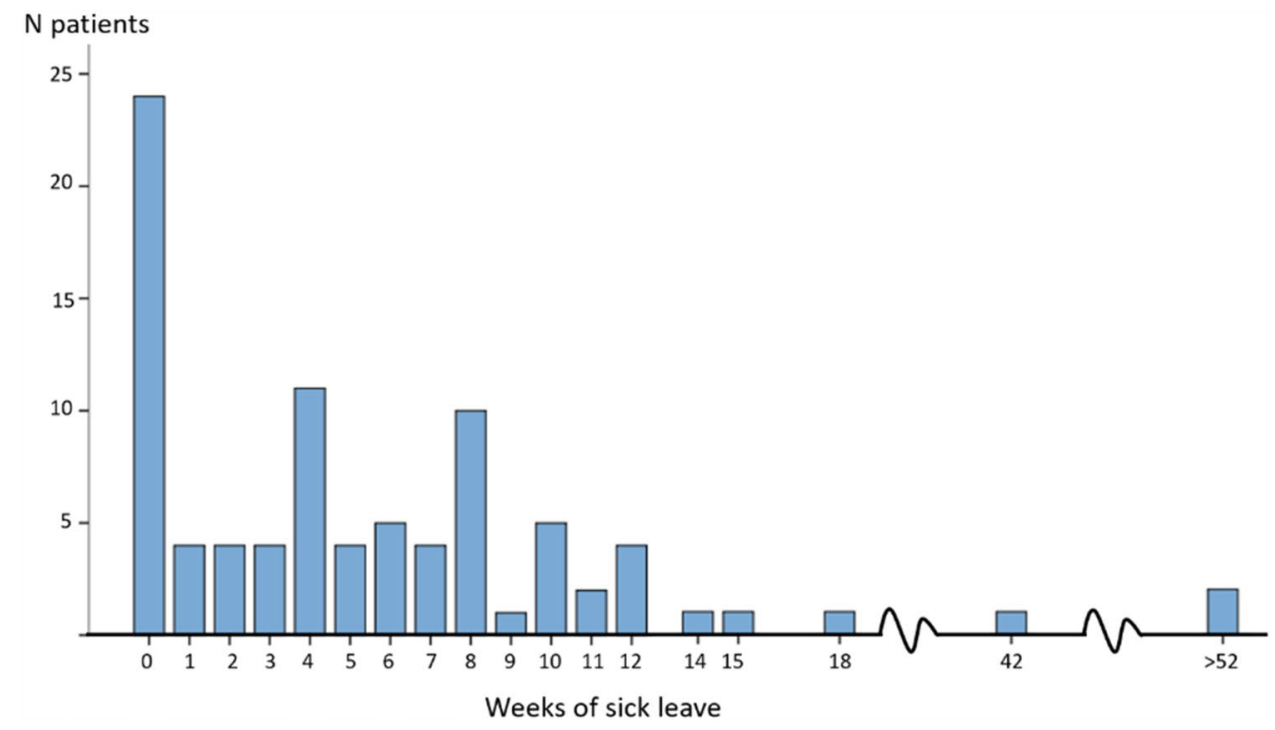

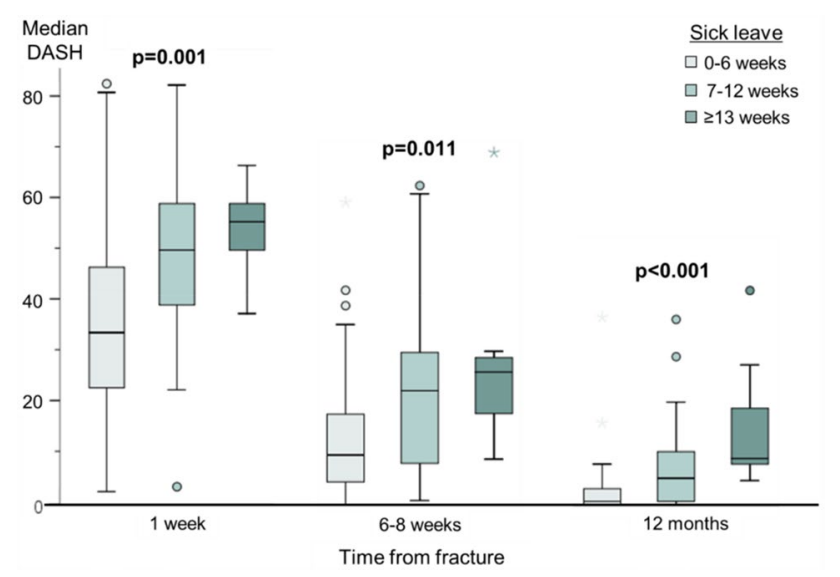

Fig. 2 Self-reported disability (DASH score) at 1 week, 6-8 weeks and 12 months by sick leave category. Reported values are median DASH score and 95\% Confidence Intervals

between length of sick leave and DASH score, intensity of pain and PCS was apparent already at one week after fracture. The correlation between sick leave and pain at one week was even stronger when analyzing treatment groups separately (closed reduction and cast $\mathrm{r}_{\mathrm{s}}=0.56, \mathrm{p}=0.007$, surgery $\left.\mathrm{r}_{\mathrm{s}}=0.42, \mathrm{p}=0.04\right)$. There was no correlation between global mental health MCS and sick leave at any time point.

Conventionally evaluated factors e.g. treatment method and radiographic measurements generally showed weaker association to length of sick leave (Table 2). Age was correlated to sick leave as well as educational level, although the effect of the latter was diminished when adjusting for work demand.

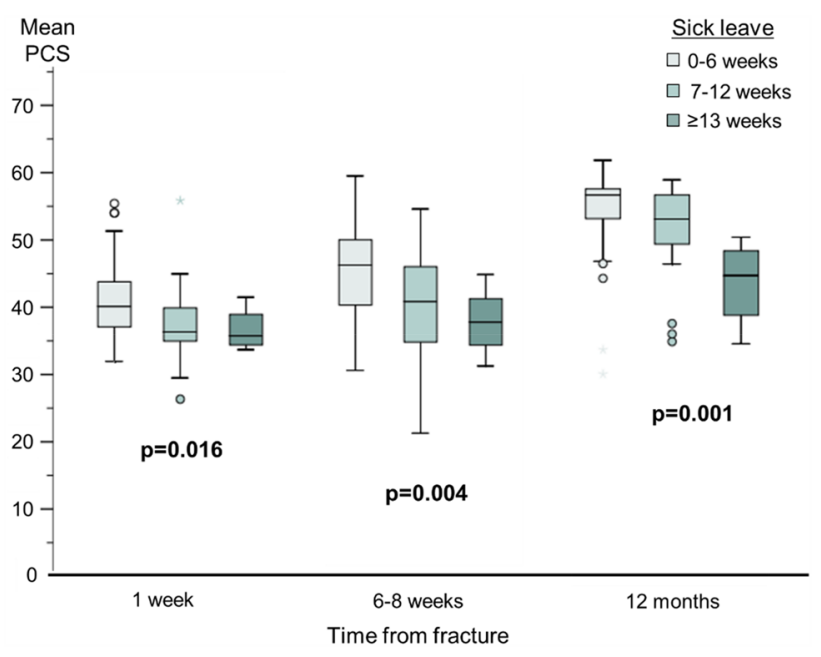

Fig. 3 Self-reported global health (SF-36 PCS) at 1 week, 6-8 weeks and 12 months by sick leave category. Reported values are mean SF-36 Physical Component Score (PCS) and 95\% Confidence Intervals

Fracture severity at the initial presentation, measured as radiographic displacement (dorsal tilt $>10^{\circ}$ and/or ulnar variance $>2 \mathrm{~mm}$ ) and treatment type were associated with sick leave. Of the radiographic parameters only dorsal tilt at initial presentation correlated with sick leave $\left(\mathrm{r}_{\mathrm{s}}=-0.3\right.$, $\mathrm{p}=0.02$ ). Ulnar variance or dorsal tilt at follow-up evaluation were not associated. Neither trauma level nor involvement of the dominant hand correlated with length of sick leave.

Finally, to identify potential predictors of length of sick leave, we performed stepwise multiple linear regression analysis (Table 3). The ground model, which included nonfracture related factors i.e. age, CCI and work demand, 
Table 2 Correlations, $r_{s}$ with $95 \%$ CI, between duration of sick leave and self-reported disability/global health and patient/fracture related factors

Spearmans $\mathrm{r}_{\mathrm{s}}(95 \% \mathrm{CI}) \quad p$-value

\begin{tabular}{|c|c|c|c|}
\hline \multicolumn{4}{|l|}{ A) Disability/global health } \\
\hline \multicolumn{4}{|l|}{1 week } \\
\hline DASH score & 0.38 & $(0.19,0.55)$ & $<0.001$ \\
\hline DASH Pain & 0.41 & $(0.22,0.57)$ & $<0.001$ \\
\hline SF-36 PCS ${ }^{\mathrm{a}}$ & -0.35 & $(-0.53,-0.14)$ & 0.002 \\
\hline SF-36 MCS ${ }^{b}$ & -0.05 & $(-0.27,0.18)$ & 0.687 \\
\hline \multicolumn{4}{|l|}{$6-8$ weeks } \\
\hline DASH score & 0.38 & $(0.17,0.56)$ & 0.001 \\
\hline DASH Pain & 0.37 & $(0.16,0.55)$ & 0.001 \\
\hline SF-36 PCS & -0.41 & $(-0.58,-0.21)$ & $<0.001$ \\
\hline SF-36 MCS & -0.08 & $(-0.39,0.15)$ & 0.516 \\
\hline EQ5D VAS & -0.30 & $(-0.50,-0.075)$ & 0.011 \\
\hline \multicolumn{4}{|l|}{12 months } \\
\hline DASH score & 0.52 & $(0.32,0.67)$ & $<0.001$ \\
\hline DASH Pain & 0.30 & $(0.075,0.50)$ & 0.010 \\
\hline SF-36 PCS & -0.46 & $(-0.63,-0.25)$ & $<0.001$ \\
\hline SF-36 MCS & 0.03 & $(-0.21,0.27)$ & 0.785 \\
\hline EQ5D VAS & -0.34 & $(-0.53,-0.12)$ & 0.004 \\
\hline \multicolumn{4}{|l|}{ B) Patient related factors } \\
\hline Age & 0.31 & $(0.11,0.49)$ & 0.004 \\
\hline $\mathrm{CCI}$ & 0.03 & $(-0.18,0.24)$ & 0.779 \\
\hline Alcohol, units/week & -0.14 & $(-0.35,0.084)$ & 0.237 \\
\hline Educational level & -0.36 & $(-0.53,-0.16)$ & 0.001 \\
\hline Work demand ${ }^{\mathrm{c}}$ & 0.28 & $(0.071,0.47)$ & 0.008 \\
\hline \multicolumn{4}{|l|}{ C) Fracture related factors } \\
\hline Trauma level & 0.0003 & $3(-0.21,0.21)$ & 0.974 \\
\hline Dominant hand fracture & -0.21 & $(-0.42,0.018)$ & 0.077 \\
\hline Displacement $^{\mathrm{d}}$ initial & 0.27 & $(0.046,0.47)$ & 0.021 \\
\hline $\begin{array}{l}\text { Displacement }{ }^{\mathrm{d}} \\
\text { follow-up }\end{array}$ & 0.028 & $(-0.20,0.25)$ & 0.809 \\
\hline Treatment $\mathrm{e}^{\mathrm{e}}$ & 0.29 & $(0.086,0.47)$ & 0.006 \\
\hline Complication-major & 0.078 & $(-0.13,0.28)$ & 0.471 \\
\hline
\end{tabular}

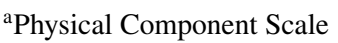

${ }^{\mathrm{b}}$ Mental Component Scale

${ }^{c}$ Dichotomized into light-medium/high-very high

${ }^{\mathrm{d}}$ Dorsal tilt $>10^{\circ}$ and/or ulnar variance $>2 \mathrm{~mm}$

${ }^{\mathrm{e}}$ Treatment categories: cast, closed reduction and cast, surgery

explained $19 \%$ of the variation in length of sick leave. The addition of disability, intensity of pain or perception of global health at either one or 6-8 weeks after fracture to this model increased prediction to $23-27 \%$.

Recognizing that fracture severity and hence type of treatment influence rehabilitation, model 2 included treatment method which then explained $28 \%$ of the variation in sick leave. The addition of self-reported measures of disability and global health i.e. 1-week DASH score or intensity of pain added significantly to prediction, now explaining $32 \%$ and $35 \%$ of sick leave, increasing to $35 \%$ and $37 \%$ ) at $6-8$ weeks.

\section{Discussion}

In the present study, we find that sick leave after a distal radius fracture in men of working age is highly variable. Almost one quarter of men with radius fracture do not lose any time from work and few are unable to return to work 12 months after fracture. The most consistent finding was the impact of self-reported disability and physical health on length of sick leave and most interestingly, the early prediction of prolonged sick leave by the intensity of pain.

The only previous study on the subject is by Macdermid et al. [4] and we find both differences and similarities. The cohorts are comparable regarding age, dominant hand fracture, prevalence of surgery and work demand,however, although accounting for forty percent of their cohort, men were not analyzed separately. There is a quite large difference in length of sick leave following distal radius fracture between the studies; Macdermid found a median 8 weeks sick leave compared to only 4 weeks in our male only cohort. Whether this finding is being skewed by including women or if there is an impact of differences in social insurance systems is difficult to judge. Participants in the Macdermid study exhibited much worse DASH scores at three and twelve months, although the work loss categories are not quite the same and notably, it is unclear whether median or mean DASH scores are reported. However, both studies share the main conclusions: that approximately twenty percent do not lose any time off work and that self-reported disability is the strongest predictor of length of sick leave.

A finding of greater importance is that higher disability and pain intensity as early as 1 week after a distal radius fracture, is a risk factor for longer sick leave in men, whether treated non-surgically or surgically. This is supported by an earlier study, showing that baseline pain intensity predicts chronic pain in both men and women with distal radius fracture [17].

The subjective symptom "pain" is multifactorial involving both injury (fracture severity, treatment, complications) and patient related factors (age, educational level, prior pain experience and psychosocial factors) [18]. Many of these factors are nonmodifiable but should be considered,early identification of risk factors leading to chronic musculoskeletal pain following an acute injury may minimize the risk of persistent pain, disability and prolonged sick leave. There is still no consensus on the most appropriate rehabilitation program, supervised therapy versus a home exercise program, following a distal 
Table 3 Predictors of length sick leave after distal radius fracture

\begin{tabular}{lllll}
\hline & $R^{2}$ & $\mathrm{~B}[95 \% \mathrm{CI}]$ & $R^{2}$ & $\mathrm{~B}[95 \% \mathrm{CI}]$ \\
\hline $\begin{array}{l}\text { Model 1 } \\
\text { Age }\end{array}$ & 0.19 & $0.12[0.043,0.19]$ & & \\
CCI $^{\mathrm{a}}$ & & $-0.60[-2.11,0.94]$ & & \\
Work demand $^{\mathrm{b}}$ & & $3.96[1.56,6.37]$ & & \\
& 1 week & & $6-8$ weeks & $1.075[0.005,0.15]$ \\
DASH score & 0.25 & $0.050[0.003,0.096]$ & 0.23 & $-0.20[-0.33,-0.070]$ \\
DASH Pain & 0.27 & $1.18[0.15,2.22]$ & 0.25 & \\
SF-36 PCS & 0.23 & $-0.12[-0.28,0.031]$ & 0.27 & \\
Model 2 & & & & \\
Age & 0.28 & $0.086[0.018,0.15]$ & & \\
CCI & & $-0.68[-2.09,0.73]$ & & \\
Work demand & & $4.01[1.76,6.27]$ & & $0.062[-0.006,0.13]$ \\
Treatment & & $1.47[0.43,2.51]$ & & $1.036[-0.020,2.09]$ \\
& & & $6-8$ weeks & \\
DASH score & 0.32 & $0.036[-0.010,0.082]$ & 0.34 & $-0.21[-0.33,-0.085]$ \\
DASH Pain & 0.35 & $0.96[-0.035,1.96]$ & 0.35 & \\
SF-36 PCS & 0.32 & $-0.095[-0.25,0.057]$ & 0.37 & \\
\hline
\end{tabular}

The stepwise multiple regression relies on the ground model including age, $\mathrm{CCI} \dagger$ and work demand $\ddagger$ (model 1) or with treatment (model 2) and separately adding self-reported disability, pain or physical health. Data are presented as $\mathrm{R}^{2}$ and unstandardized regressions coefficient, $\mathrm{B}$, with $95 \% \mathrm{CI}$

${ }^{a}$ Charleson Comorbidity Index

${ }^{\mathrm{b}}$ Dichotomized into light-medium/high-very high

${ }^{\mathrm{c}}$ Treatment in three groups: cast, closed reduction and cast, surgery radius fracture in adults [19] and current recommendation emphasize clinicians to rely on their experience and clinical judgement [20]. Our study indicates, that a higher degree of pain at an early stage, regardless of treatment type, is predictive of longer sick leave and as a yellow flag assist the clinician in allocating rehabilitation resources and other pain-reducing treatment.

We found that higher age at fracture was associated with longer sick leave which contrasts with the Macdermid study [4]. Lower education is a known risk factor for unhealthy life style and sick leave [21, 22]. In the present study, however, the association between sick leave and educational level was diminished when adjusting for work demand since work demand was higher in those with lower education. Remarkably, we didn't find the strong association between work demand and sick leave as would be expected.

On the other hand, we find that aspects traditionally regarded as important, such as fracture of the dominant hand, fracture severity measured by classification and displacement at follow-up, were not associated with longer sick leave. However, only a few had displacement at follow-up, which might preclude statistical significance. High trauma level in the non-osteoporotic population has been hypothesized to correlate to more severe soft tissue injury [23],we did not find any correlation between trauma level and sick leave. At the time of this investigation surgery was primarily external fixation for 5 weeks, while presently the current trend favors volar plates as the treatment of choice, which may result in shorter length of sick leave due to earlier mobilization [24].

The main strengths of this study are the prospective design, the continuous follow-up and most importantly, the explicit focus on men, who have been excluded from or comprised a minority in many prior studies. In contrast to most of the current research which concentrates on fracture severity and treatment methods, we focus on selfreported outcome measurements in addition to traditionally evaluated factors providing further insight regarding sick leave after distal radius fracture in men. It also becomes clear that larger clinical investigations are warranted to address the increasing prevalence of distal radius fracture in the expanding working-age population and to improve identification of individuals at risk of long sick leave.

We recognize several limitations. Firstly, the cohort is relatively small and hence may be underpowered to detect differences especially in those groups with low numbers, i.e. participants with prolonged sick leave and displacement at follow-up. There is also the risk of selection bias; however, we found no difference in age distribution between participants and non-participants. This cohort is part of a study on osteoporosis in men with distal radius fracture. Our participation rate is similar to equivalent osteoporosis 
studies, but different from mail-based studies focusing only on self-reported outcome $[25,26]$ with physical participation being more demanding than returning questionnaires. Furthermore, studies in men often have lower participation rates [25-27]. Thirdly, we did not have objective functional measurements such as range of motion or grip strength, which could have been valuable. On the other hand, these variables have not been shown to clearly correlate with disability in terms of DASH scores [28]. Fourthly, our sick leave data was self-reported, which may not fully agree with administrative data [29, 30]. Recall bias can be expected, but we believe that the continuous follow-up reduces such impact.

Finally, we recognize the complexity of sick leave after a fracture. Although this study was based on prospectively collected data, the research question was designed after conclusion of the study. Thus, not all potentially interesting predictors were included (work culture, social situation and the individual's sense of coherence). However, this was not within the scope of this study and we focus on factors readily available in daily clinical management, aware that we are missing important pieces of the puzzle.

We conclude that for men of working age with distal radius fracture, although duration of sick leave is highly variable, those with higher perceived disability and pain as early as 1 week post-fracture have longer sick leave, a finding that should influence clinical management directly. Furthermore, patient-reported measures are more predictive of length of sick leave than traditional clinical variables such as radiographic presentation and should be incorporated in the standard management.

Acknowledgements Open access funding provided by Lund University. Thanks are extended to Jan-Åke Nilsson for statistical advice.

Author Contributions All authors contributed to the study conception and design. Material preparation, data collection and analysis were performed by LE, KÖ and KA. The first draft of the manuscript was written by LE and all authors commented on previous versions of the manuscript. All authors read and approved the final manuscript.

Funding This work was supported by the Swedish Research Council (K2015-52X-14691-13-4), Greta and Johan Kock Foundation, A. Påhlsson Foundation, A. Osterlund Foundation, H Järnhardt foundation, Skåne University Hospital Research Fund and the Research and Development Council of Region Skåne, Sweden.

\section{Compliance with Ethical Standards}

Conflict of interest The authors, Lisa Egund, Karin Önnby, Fiona Mcguigan and Kristina Åkesson declare no potential conflicts of interest with respect to the research, authorship, and/or publication of this article.

Ethical Approval All procedures followed were in accordance with the ethical standards of the responsible committee on human experimentation (institutional and national) and with the Helsinki Declaration of 1975, as revised in 2000.

Informed Consent Informed consent was obtained from all patients for being included in the study.

Open Access This article is licensed under a Creative Commons Attribution 4.0 International License, which permits use, sharing, adaptation, distribution and reproduction in any medium or format, as long as you give appropriate credit to the original author(s) and the source, provide a link to the Creative Commons licence, and indicate if changes were made. The images or other third party material in this article are included in the article's Creative Commons licence, unless indicated otherwise in a credit line to the material. If material is not included in the article's Creative Commons licence and your intended use is not permitted by statutory regulation or exceeds the permitted use, you will need to obtain permission directly from the copyright holder. To view a copy of this licence, visit http://creativecommons .org/licenses/by/4.0/.

\section{References}

1. Jerrhag D, Englund M, Karlsson MK, Rosengren BE. Epidemiology and time trends of distal forearm fractures in adults-a study of 11.2 million person-years in Sweden. BMC Musculoskelet Disord. 2017. https://doi.org/10.1186/s1289 1-017-1596-zs.

2. Brogren E, Petranek M, Atroshi I. Incidence and characteristics of distal radius fractures in a southern Swedish region. BMC Musculoskelet Disord. 2007;8(1):48.

3. Brogren E, Hofer M, Petranek M, Wagner P, Dahlin LB, Atroshi I. Relationship between distal radius fracture malunion and armrelated disability: a prospective population-based cohort study with 1-year follow-up. BMC Musculoskelet Disord. 2011;12:9.

4. MacDermid JC, Roth JH, McMurtry R. Predictors of Time Lost from Work Following a Distal Radius Fracture. J Occup Rehabil. 2007;17(1):47-62.

5. Kraus TM, Freude T, Fiedler S, Schröter S, Stöckle U, Ateschrang A. Incapacity of work after arthroscopic Bankart repair. Arch Orthop Trauma Surg. 2015;135(10):1429-36.

6. Kraus TM, Abele C, Freude T, Ateschrang A, Stöckle U, Stuby FM, et al. Duration of incapacity of work after tibial plateau fracture is affected by work intensity. BMC Musculoskelet Disord. 2018. https://doi.org/10.1186/s12891-018-2209-1.

7. Egund L, McGuigan F, Önnby K, Giwercman A, Akesson K. High prevalence of osteoporosis in men with distal radius fracture: a cross-sectional study of 233 men. Calcif Tissue Int. 2016;99(3):250-8.

8. Abramo A, Kopylov P, Tagil M. Evaluation of a treatment protocol in distal radius fractures: a prospective study in 581 patients using DASH as outcome. Acta Orthop. 2008;79(3):376-85.

9. OALJ Law Library, Dictionary of Occupational Titles, Appendix C-Office of Administrative Law Judges, Washington (1991). https://www.lb7.uscourts.gov/documents/INND/310CV4272.PDF

10. Hudak PL, Amadio PC, Bombardier C. Development of an upper extremity outcome measure: the DASH (disabilities of the arm, shoulder and hand). The Upper Extremity Collaborative Group (UECG). Am J Ind Med. 1996;29(6):602-8.

11. Ware JE. SF-36 health survey update. Spine. 2000;25(24):3130-9.

12. Rabin R, Gudex C, Selai C, Herdman M. From translation to version management: a history and review of methods for the cultural adaptation of the EuroQol five-dimensional questionnaire. Value Health. 2014;17(1):70-6. 
13. Goldfarb CA, Yin Y, Gilula LA, Fisher AJ, Boyer MI. Wrist fractures: what the clinician wants to know. Radiology. 2001;219(1):11-28.

14. Steyers CM, Blair WF. Measuring ulnar variance: a comparison of techniques. J Hand Surg Am. 1989;14(4):607-12.

15. Slutsky DJ, Osterman AL. Fractures and injuries of the distal radius and carpus: the cutting edge. Amsterdam: Elsevier Health Sciences; 2009.

16. Ramel E, Rosberg H-E, Dahlin LB, Cederlund RI. Return to work after a serious hand injury. Work. 2013;44(4):459-69.

17. Mehta SP, MacDermid JC, Richardson J, MacIntyre NJ, Grewal $\mathrm{R}$. Baseline pain intensity is a predictor of chronic pain in individuals with distal radius fracture. J Orthop Sports Phys Ther. 2015;45(2):119-27.

18. Mehta S, MacDermid J, Tremblay M. The implications of chronic pain models for rehabilitation of distal radius fracture. Hand Therapy. 2011;16(1):2-11.

19. Valdes K, Naughton N, Michlovitz S. Therapist supervised clinic-based therapy versus instruction in a home program following distal radius fracture: a systematic review. J Hand Ther. 2014;27(3):165-73.

20. Lucado AM. Clinical commentary: therapist supervised clinicbased therapy versus instruction in a home program following distal radius fracture: a systematic review. J Hand Ther. 2014;27(3):175-6.

21. Fuhrer R, Shipley MJ, Chastang JF, Schmaus A, Niedhammer I, Stansfeld SA, et al. Socioeconomic position, health, and possible explanations: a tale of two cohorts. Am J Public Health. 2002;92(8):1290-4.

22. Marmot M, Ryff CD, Bumpass LL, Shipley M, Marks NF. Social inequalities in health: next questions and converging evidence. Soc Sci Med. 1997;44(6):901-10.

23. Lindau T. Arthroscopic evaluation of associated soft tissue injuries in distal radius fractures. Hand Clin. 2017;33(4):651-8.
24. Abramo A, Kopylov P, Geijer M, Tägil M. Open reduction and internal fixation compared to closed reduction and external fixation in distal radial fractures. Acta Orthop. 2009;80(4):478-85.

25. Øyen J, Gjesdal CG, Brudvik C, Hove LM, Apalset EM, Gulseth $\mathrm{HC}$, et al. Low-energy distal radius fractures in middle-aged and elderly men and women-the burden of osteoporosis and fracture risk: a study of 1794 consecutive patients. Osteoporos Int. 2010;21(7):1257-67.

26. Landgren M, Abramo A, Geijer M, Kopylov P, Tägil M. Similar 1-year subjective outcome after a distal radius fracture during the 10-year-period 2003-2012. Acta Orthop. 2017;88(4):451-6.

27. Mindell JS, Giampaoli S, Goesswald A, Kamtsiuris P, Mann C, Männistö S, et al. Sample selection, recruitment and participation rates in health examination surveys in Europe-experience from seven national surveys. BMC Med Res Methodol [Internet]. 2015 Oct 5;15. https://www.ncbi.nlm.nih.gov/pmc/articles/PMC45 95185/. Accessed 24 Sep 2018

28. Karnezis IA, Fragkiadakis EG. Association between objective clinical variables and patient-rated disability of the wrist. J Bone Joint Surg Br. 2002;84(7):967-70.

29. Pole JD, Franche R-L, Hogg-Johnson S, Vidmar M, Krause N. Duration of work disability: a comparison of self-report and administrative data. Am J Ind Med. 2006;49(5):394-401.

30. Dasinger LK, Krause N, Deegan LJ, Brand RJ, Rudolph L. Duration of work disability after low back injury: a comparison of administrative and self-reported outcomes. Am J Ind Med. 1999;35(6):619-31.

Publisher's Note Springer Nature remains neutral with regard to jurisdictional claims in published maps and institutional affiliations. 